\title{
Unmittelbare Remission eines mit großzelligem B-Non-Hodgkin-Lymphom befallenen inguinalen Lymphknotens unter alleiniger homöopathischer Behandlung mit Conium: Wann ist eine alleinige adjuvant-homöopathische Tumortherapie zulässig und sinnvoll?
}

\author{
Heinrich Hümmer Timm Wiecken Katharina Pachmann \\ Praxis für Allgemeinmedizin Dr. Wiecken - Dr. Wolf - Dr. Hümmer, Herrsching, Deutschland
}

Schlüsselwörter

B-Zell-Non-Hodgkin-Lymphom · Adjuvante

homöopathische Tumor-Therapie · Conium · Remission

\section{Zusammenfassung}

Bei einer 63-jährigen Patientin wird mittels Biopsie eines linksinguinalen Lymphknotens ein großzelliges B-NonHodgkin-Lymphom diagnostiziert. Unmittelbar nach Beginn einer homöopathischen Therapie mit Conium $\mathrm{C} 30$ beginnt sich der Lymphknoten in der linken Leiste zurückzubilden. Bei Exzision des Lymphknotens vierzehn Tage nach Therapiebeginn können histologisch keine Residuen des Tumors mehr nachgewiesen werden und es darf von einer vollständigen Remission ausgegangen werden. Die Patientin bleibt in der Folge rezidivfrei. Das homöopathische Mittel Conium (Schierling) kommt in der adjuvanten homöopathischen Tumortherapie und bei vergrößerten Lymphknoten als häufig indiziertes Mittel zur Anwendung.

(c) 2019 The Author(s)

Published by S. Karger AG, Basel

Patientin K.A. ist der Redaktion bekannt und bestätigt die Darstellung des Krankheitsverlaufs und sämtlicher erhobener Befunde.
Immediate Remission of an Inguinal Lymph Node Afflicted with Large-Cell B-Non-Hodgkin's Lymphoma Under Sole Homeopathic Treatment with Conium: When Is a Sole Adjuvant-Homeopathic Tumor Therapy Permissible and Useful?

\section{Keywords}

B-Cell Non-Hodgkin Lymphoma · Adjuvant homeopathic tumor therapy $\cdot$ Conium $\cdot$ Remission

\section{Abstract}

A large-cell B-cell non-Hodgkin Lymphoma ( $L C B C L)$ was diagnosed bioptically in a female patient (age 63 years) in one left inguinal lymph node. Immediately after beginning homeopathic treatment with Conium C 30, the lymph node started to show a reduction in size. Two weeks after starting homeopathic therapy, histological examination of the excised lymph node showed no evidence of a residual tumor - suggestive of a complete remission. The patient remains disease free until now. The homeopathic remedy Conium (hemlock) is frequently applied for adjuvant homeopathic tumor therapy as well as for the treatment of enlarged lymph nodes. @ 2019 The Author(s)

Published by S. Karger AG, Basel

\begin{tabular}{ll}
\hline KARGER & $\begin{array}{l}\text { ( ) } 2019 \text { The Author(s) } \\
\text { Published by S. Karger AG, Basel }\end{array}$ \\
E-Mail karger@karger.com & $\begin{array}{l}\text { This article is licensed under the Creative Commons Attribution- } \\
\text { www.karger.com/cmr }\end{array}$ \\
$\begin{array}{l}\text { NonCommercial-NoDerivatives 4.0 International License (CC BY- } \\
\text { NC-ND) (http://www.karger.com/Services/OpenAccessLicense). } \\
\text { Usage and distribution for commercial purposes as well as any dis- } \\
\text { tribution of modified material requires written permission }\end{array}$
\end{tabular}




\section{Falldarstellung}

Am 25.5.2018 stellt sich die 63-jährige Patientin K.A. nach längerer Abwesenheit von der Praxis vor wegen eines von ihr seit 4 Wochen bemerkten vergrößerten Lymphknotens in der linken Leiste. Abgesehen von einem Spannungsgefühl im Bereich des Lymphknotens werden keinerlei lokale oder abdominelle Beschwerden angegeben. Sie gibt an, sich seit dem letzten Besuch am 5.7.2005 in der Praxis völlig gesund gefühlt zu haben, ohne die Erfordernis jeglicher Arztbesuche. Es bestehen keine Stuhlgangsauffälligkeiten oder B-Symptomatik. Eine gynäkologische Untersuchung erfolgte zuletzt am 11.7.2006 mit einem unauffälligen Zervixabstrich.

Die Patientin ist seit 1991 wegen grippaler Infekte und anderer interkurrenter Erkrankungen in hiesiger hausärztlicher konventionellen, naturheilkundlichen und homöopathischen Behandlung. Eine deutliche Reaktion im Sinne einer sogenannten homöopathischen Erstverstärkung und anschließend Besserung der Symptome zeigte sie nach homöopathischer Gabe von Conium (Schierling) in der Potenzierungsstufe C 30 im Juni 2005, die sie wegen allgemeinen Erschöpfungsgefühls bei gynäkologischerseits diagnostizierter Zervixdysplasie erhielt.

Die klinische Untersuchung ergibt bis auf einen derben, nicht druckdolenten, unverschieblichen, auf Kirschgröße vergrößerten Lymphknoten in der linken Leiste keine pathologischen Befunde und insbesondere keine weiteren Lymphknotenvergrößerungen (Hals, Axilla, supraclavikulär).

Die ebenfalls am 25.5.2018 durchgeführte sonographische Begutachtung des Lymphknotens ergibt einen auf max. $2,7 \mathrm{~cm}$ vergrößerten, echoarmen und kräftig vaskularisierten Lymphknoten mit gut abgrenzbarem Hilus, jedoch fehlendem Hiluszeichen. Somit findet sich eine suspekte Morphologie. Die sonstigen abdominellen Organe zeigen sonographisch bis auf ein fragliches LeberHämangiom keine pathologischen Veränderungen.

Bei dringendem Verdacht auf einen pathologisch vergrößerten Lymphknoten umfasst das Procedere zunächst Laborparameterüberprüfungen, Computertomographie von Thorax und Abdomen, Coloskopie, gynäkologische Untersuchung, Lymphknoten-Stanzbiopsie und Vorstellung beim Onkologen. Im Labor zeigt sich lediglich eine mäßige Erhöhung der Transaminasen GOT, GPT und Gamma-GT bei unauffälliger Hepatitis A-, B-, C- und Lyme-Serologie.

Bei der am 13.6.2018 durchgeführten Computertomographie von Thorax und Abdomen kommen bei ansonsten unauffälligen Befunden in der linken Leiste mehrere vergrößerte Lymphknoten zur Darstellung, wobei der größte bereits einen Durchmesser von ca. $4 \mathrm{~cm}$ aufweist. Mehrere weitere vergrößerte Lymphknoten bis zu 1,3 cm Durchmesser sind unterhalb des klinisch auffälligen Lymphknotens in der linken Leiste sichtbar.
Die am 11.7.2018 durchgeführte Lymphknoten-Stanzbiopsie des inzwischen auf sonographisch $4 \mathrm{~cm}$ Durchmesser angewachsenen Lymphknotens ergibt den in $\mathrm{Ab}$ bildung 1 wiedergegebenen histopathologischen und histochemisch bestätigten Befund eines malignen NonHodgkin-Lymphoms der B-Zell-Reihe vom Typ eines diffus großzelligen Lymphoms, nicht-KeimzentrumsTyp nach Hans.

Die Untersuchung des Knochenmarkaspirats vom 18.7.2018 ergibt keinen Hinweis auf eine Knochenmarktinfiltration durch ein Lymphom. Eine Coloskopie vom 18.6.2018 zeigt bis auf eine Divertikulose Normalbefunde bis zum terminalen Ileum. Die gynäkologische Untersuchung ist ohne pathologischen Befund.

Die Patientin beginnt eine Woche nach bioptisch gesicherter Diagnose die adjuvante homöopathische Therapie mit Conium C 30.

Nach Vorliegen aller Befunde empfiehlt die Onkologin bei Diagnose eines hoch-malignen Non-HodgkinLymphoms der B-Zellreihe eine systemische Chemotherapie mit 6 Zyklen R-CHOP (Rituximab-Cyclophosphamid-Doxorubicin-Vincristin-Prednison) in 3-wöchigen Abständen. Sie rät zudem zu einem "raschen Therapiebeginn (zur Vermeidung einer Verschlechterung der Prognose bei aktuell niedrigem Risikoprofil)."

Da die Patientin jedoch schon vor dem erstem Chemotherapie-Termin und unmittelbar nach Beginn der homöopathischen Therapie eine Verkleinerung des Lymphknotens feststellt, rät die Onkologin nach Rücksprache mit der Praxis zur chirurgischen Exstirpation des suspekten Lymphknotens zur Sicherung der vorangegangenen bioptischen Ergebnisse.

\section{Adjuvante homöopathische Therapie}

Mit der Patientin war 1991 bei Beginn der ersten therapeutischen Interventionen eine ausführliche homöopathische Erstanamnese durchgeführt worden, welche einen Hinweis auf Conium als sogenanntes Konstitutionsmittel ergeben hatte. Sie hatte zudem zwischenzeitlich bei Auftreten eines benignen, paroxysmalen Lageschwindels und bei einem Fatigue-Syndrom im Rahmen einer Zervixdysplasie positiv auf eine homöopathische Therapie mit Conium angesprochen. Unter Berücksichtigung der Tumorund Lymphknoten-Rubriken im Repertorium Synthesis $[1,2]$, die jetzt erneut auf Conium als angezeigtes Mittel hinweisen, wird der Patientin Conium zur täglichen Einnahme empfohlen.

Sie beginnt ab 18.7.2018 mit der Einnahme von täglich 1 Granulum Conium C 30 von der Firma Homeoden/Belgien. Bereits am Folgetag bemerkt sie eine Abnahme des Spannungsgefühls im Bereich des vergrößerten linksinguinalen Lymphknotens und in den nächsten Tagen eine eindeutige, stetige Größenabnahme des Lymphknotens.

Nachdem sich der subjektive Eindruck der Patientin in der klinischen Untersuchung bestätigt, wird für sie am 25.7.2018 zur externen Überprüfung der Größenveränderung des inguinalen Lymphknotens eine erneute sonographische Begutachtung durch die örtliche Radiologie veranlasst und am selben Tag durchge- 
Erneuter Ausdruck des Befundes mit Zusatzbericht.

Art/Herkunft des Materials

Stanzbiopsie Lymphknoten links inguinal

Histopathologische Begutachtung:

Mikroskopie:

Drei hintereinandergelegt $2,2 \mathrm{~cm}$ lange Stanzbiopsiezylincer aus sehr dichtgelagerten Zellen mit relativ großen teils vesikulären Zellkernen und aberwlegend mehreren prominenten Nukleolen. Eingestreut kleinkernige Lymphozyten. Keine Follikelstruktur erkennbar.

Beurteilung:

Stanzbiopsien aus einem Lymphknoten mit einem atypischen Zellinfiltrat, vereinbar mit einem malignen Lymphom.

Zur weiteren Klassifikation sind immunhistochemischen Färbungen erforderlich. Hierzu folgt ein Zusatzbericht.

PD Dr. H. Nagel

(Interne Zweltbeurteilung im Rahmen des Qualitätsmanagements durch PC Dr. W. Maller)

Bericht per EDV als Telefax aufbereitet und daher nicht unterschrieben

\section{Zusatzbericht}

Inzwischen fiegen immunhistochemische Untersuchungen vor. Das atypische Zellinfiltrat konstant kräftig positiv für CD20 und BCL2 ohne erkennbare Follikelstrukturen. Keine Koexpression von CD10 und keine Expression von Cyclin D1 in den Tumorzellen. Ki67 wird von etwa $90 \%$ der Zellen exprimlert.

\section{Beurtellung:}

Stanzbiopsien mit einem aggressiven Non-Hodgkin Lymphom vom Typ eines diffusen großzelligen B-Zell-Lymphoms. Zur weiteren Subklassifikation erfolgen noch zusatzliche immunhistochemische Untersuchungen und ein emeuter Zusatzbericht.

PD Dr. H. Nagel

(Interne Zweitbeurteilung im Rahmen des Qualitatsmanagements durch PD Dr. W. Maller)

Entsprechend den gesetzlichen Vorgaben wurde der Befund an das Tumorregister München gemaldet. Wir bitten darum, die Patientin auf ihr Wderspruchsrecht hinzuweisen.

Bericht per EDV als Telefax autbereitet und daher nicht unterschirioben

\section{Zusatzbericht}

Inzwischen wurden weitere immunhistochemische Untersuchungen durchgefahrt. Etwa $50 \%$ der Tumorzellen zeigon eino krättige nukleăro Anfärbung für MUM1 und mehr als $80 \%$ dieser Zellen sind positiv für BCL6.

Beurteilung:

Malignes Non Hodgkin-Lymphom der B-Zell-Roiho vom Typ eines diffusen großzelrigen Lymphoms, nicht-Koimzentrums-Typ nach Hans.

Abb. 1. Histopathologischer Befund Stanzbiopsie linksinguinaler Lymphknoten. 
Erneuter Ausdruck des Befundes mit Zusatzbericht.

Art/Herkunft des Materials

Lymphknoten linke Leiste

Histopathologische Begutachtung:

Makroskopie:

Bohnenförmiger fettbehafteter Lymphknoten von 3,5 x 1,8 x 1,5 cm. Die Schnittfläche homogen hellbraun.

(Entnahme f. Histologie: zentraler QS).

Mikroskopie:

Anschnitte durch einen vergrößerten Lymphknoten dessen Stroma abschnittsweise fibrosiert ist. Es finden sich hier teilweise Siderophagen hyalinisierte Abschnitte außen übergehend in residuelle Anteile eines Lymphknoten mit teils noch erhaltener Lymphknotenarchitektur. An der Innenseite teilweise fibrinoide Nekrosen. Außen reifzelliges Fettgewebe. Keine Befunderweiterung bei PAS- und Giemsa-Färbung.

Beurteilung:

Leistenlymphknoten mit abschnittsweise regressiver Stromafibrose (nach vorausgegangener Stanzbiopsie, vgl. H 2018/42006).

Die Veränderungen im bisher vorliegenden Material entsprechen regressiven Veränderungen (Therapiefolge?). Auch bei erneuter Durchsicht der vorausgegangenen Stanzbiopsien besteht an der primären Lymphomdiagnose kein Zweifel. Das in der Stanzbiopsie evidente Lymphom ist jedoch im bisher vorliegenden Material nicht mehr nachweisbar. Es wird noch weiteres Material eingebettet. Nach Auswertung folgt ein Nachbericht.

PD Dr. Chr. Brinkschmidt

Bericht per EDV als Telefax aufbereitet und daher nicht unterschrieben

Zusatzbericht

Auch nach kompletter Einbettung des Restmaterials ergeben sich gegenüber der Erstbefundung keine neuen Aspekte. Eindeutige Residuen des stanzbioptisch vordiagnostizierten großzellig diffusen B-Zell-Lymphoms sind weiterhin nicht nachweisbar.

PD Dr. Chr. Brinkschmidt

Abb. 2. Histopathologischer Befund exzidierter Lymphknoten linke Leiste.

führt, welche die deutliche Verkleinerung auf jetzt $28 \times 12 \mathrm{~mm}$ von zuvor über $4 \mathrm{~cm}$ Durchmesser bestätigt.

Angesichts der klinisch und sonographisch eindeutig feststellbaren Größenreduktion des pathologischen Lymphknotens erfolgt nach oben erwähnter Rücksprache mit der Onkologin die Empfehlung zur Lymphknoten-Exstirpation, um das weitere onkologische Procedere planen zu können.

Am 1.8.2018 erfolgt in der örtlichen chirurgischen Klinik die Exzision des pathologisch vergrößerten Lymphknotens. Die histologische Aufarbeitung ergibt den in Abbildung 2 wiedergegebenen Befund einer vollständigen Remission des in den Stanzbiopsien diagnostizierten aggressiven B-Zell-Non-Hodgkin-Lymphoms.

Bis 16.9.2018 führt die Patientin die tägliche Einnahme von Conium C 30 fort, gefolgt von der Einnahme von Conium C 200 bis 26.9.2018 wegen zwischenzeitlich unspezifisch eingeschränkten Allgemeinzustandes. Bei vollständiger Beschwerdefreiheit wird die homöopathische Medikation ab diesem Zeitpunkt ganz beendet mit der Maßgabe, dass bei erneutem Auftreten von subjektiven Beschwerden die Einnahme nur dann nach letztem Schema fortgesetzt würde, wenn sich bei kurzfristig anzusetzender onkologischer Kontrolluntersuchung keinerlei Hinweise auf ein Rezidiv ergeben. Monatliche Follow-ups zur Beurteilung der homöopathischen Verlaufskriterien werden vereinbart.

Bei einer onkologischerseits angesetzten Kontroll-Computertomographie, die am 20.11.2018 erfolgt, ergeben sich keine Hinweise auf ein Rezidiv des B-Non-Hodgkin-Lymphoms; Laborkontrollen am 5.11.2018 und 12.2.2019 unter besonderer Beobachtung von $\mathrm{LDH}$ bleiben unauffällig.

Die Patientin zeigt bis dato (1.3.2019) keine subjektiven oder klinischen Anzeichen eines Rezidivs. Weitere regelmäßige und engmaschige Kontrolluntersuchungen durch die Onkologin sind vereinbart. 


\section{Diskussion}

Die homöopathische Therapie muss sich entsprechend den wissenschaftlichen und medizinischen Evidenz-Kriterien im Therapiealltag bewähren, wenn sie nicht als Placebo - oder Suggestionstherapie - eingeordnet bleiben will [3]. Sie wird dabei auch den Anforderungen hochklassiger RCTs (randomized controlled trials) genügen müssen. Bisherige Metaanalysen zur homöopathischen Studienlage kamen je nach Auswahlkriterien der zur Verfügung stehenden RCT-Studien zu unterschiedlichen Ergebnissen bezüglich der Frage, ob Homöopathie wirksamer ist als Placebo [4, 5]. Lüdtke und Rutten [6] konnten jedoch zeigen, dass die von den Kritikern als Beweis gegen die Wirksamkeit der Homöopathie am häufigsten angeführte Metastudie von Shang et al. [7] wissenschaftlich nicht belastbar ist.

Den in hoher Anzahl existierenden Einzelfall-Darstellungen homöopathischer Ärzte wurde bisher ein eher anekdotischer Charakter zugebilligt, da sie überwiegend Veränderungen subjektiver Kriterien beschrieben ohne objektive Verlaufsparameter zu liefern.

Dem gegenüber konnte der Einfluss auf objektive Tumor-Verlaufsparameter bei adjuvanter homöopathischer Tumor-Therapie bereits 2005 in einer Fallverlaufs-Studie gezeigt werden [8]. Ebenso finden sich in der neueren $\mathrm{Li}$ teratur zu homöopathischer Tumor-Therapie zahlreiche, mit engmaschiger Diagnostik abgesicherte und dokumentierte Fälle unter alleiniger homöopathischer Therapie $[9,10]$.

Einzelfallstudien aus der realen Praxis in adäquat großer Anzahl und mit ausreichender Nachbeobachtungszeit können (gerade angesichts der Probleme bei der Erstellung klinischer kontrollierter homöopathischer Studien) eine geeignete Wirknachweis-Methode darstellen.

Im hier wiedergegebenen Fall einer 63-jährigen Patientin mit bioptisch gesichertem Befall eines linksinguinalen Lymphknoten mit großzelligem B-Non-HodgkinLymphom zeigt sich unmittelbar nach Beginn einer ausschließlich homöopathischen Therapie mit Conium C 30 eine eindeutige, sonographisch objektivierbare Größenreduktion. Im weiteren Verlauf ist der Lymphknoten kaum mehr tastbar, und 2 Wochen nach Therapiebeginn finden sich im histopathologischen Präparat des exzidierten Lymphknoten bis auf "regressive Veränderungen (Therapiefolge?)" keinerlei Residuen des vordiagnostizierten großzellig diffusen B-Zell-Lymphoms.

Spontanremissionen wurden bei niedrig-malignen BZell-Non-Hodgkin-Lymphomen vereinzelt beobachtet, sind bei aggressiven, hoch-malignen Lymphomen - wie im dargestellten Fall vorliegend - allerdings äußerst selten [11]. Über einen einzelnen Fall einer spontanen Remission eines B-Non-Hodgkin-Lymphoms, das durch neuere immunhistologische Parameter als hoch-aggressiv eingeordnet werden konnte (Koexpression der Protoonkogene MYC [Myelocytomatose-Gen] und BCL2 [B-cell lymphoma 2]), wird von Potts et al. [12] berichtet.

Als auslösender Faktor für eine spontane Remission bei non-Hodgkin-Lymphomen wird vor allem die Immunmodulation durch begleitende Virusinfekte einschließlich HIV und EBV oder durch chirurgische Eingriffe diskutiert [13-17].

Auch bei sorgfältiger Literaturrecherche konnte jedoch kein Fall einer Regression nach ausschließlicher Punktion eines malignen entarteten Lymphknotens gefunden werden. In keinem der berichteten Fälle von spontaner Remission wird zudem von einer unmittelbaren Reaktion und Regression berichtet, wie sie obiger Fall erkennen lässt, noch wurde die begleitende Einnahme homöopathischer Mittel (als möglicher Einflussfaktor) explizit erfragt oder ausgeschlossen.

Die Entscheidung zur homöopathischen Therapie mit Conium (Schierling) stützte sich auf dessen Arzneimittelbild, welches den Einsatz vor allem bei Vergrößerung von Lymphknoten und adjuvant bei Tumor-Erkrankungen sowie Präkanzerosen, Fatigue-Syndrom und speziellen Formen von Schwindel beinhaltet.

Um den Beginn der onkologischerseits klar indizierten Chemotherapie nicht zu verzögern, wurde die homöopathische Therapie noch vor onkologischem Therapiebeginn angesetzt mit der absoluten Maßgabe, dass nur unmittelbare und objektive Zeichen eines Ansprechens auf die homöopathische Therapie einen eventuell verzögerten Beginn der Chemotherapie rechtfertigen. Somit konnte bereits zwei Tage nach Therapiebeginn die Entscheidung zur (zunächst vorläufigen!) Fortführung der alleinigen homöopathischen Therapie gefällt werden.

Dass bei Gabe von Conium eine Potenzierung von C 30 erfolgreich zum Einsatz kam, spricht gegen das Materie-Wirkprinzip dieser Mittelgabe, da das Mittel in einer Verdünnung beziehungsweise Potenzierung jenseits der

$$
\text { Avogadro-Konstante } \mathrm{N}_{\mathrm{A}}=6^{\prime} 022 \times 10^{23} 1 / \mathrm{mol}
$$

vorliegt, was wiederum impliziert, dass kein Molekül der Ausgangssubstanz in der Lösung vorhanden ist.

Folglich muss für diese homöopathische Mittelgabe ein Wirkprinzip jenseits des Materie-Prinzips angenommen werden. Es finden sich in der Literatur bezüglich der biologischen Wirkungen potenzierter und hochverdünnter Lösungen zunehmend hochwertige in-vitro Studien. In einer dieser Studien konnte eine Veränderung des Genexpressionsmusters humaner Nervenzellen nach Inkubation mit hochverdünnter Gelsemium-Lösung gezeigt werden [18]. 


\section{Schlussfolgerung}

Im vorliegenden Fall einer Tumorerkrankung konnte ein unmittelbares klinisches Ansprechen auf homöopathische Therapie mit einem objektivierbaren Therapieerfolg korreliert werden, der nicht plausibel durch einen Placebo-Effekt oder Suggestion erklärbar ist und der (durch den unmittelbar eintretenden Reaktionsbeginn bereits auf erste Gabe des homöopathischen Mittels) auch nicht dem Bild in der Literatur beschriebener Spontanremissionen entspricht [19]. Die beobachtete Remission ist somit auch aus onkologischer Sicht mit hoher Wahrscheinlichkeit durch die homöopathische Behandlung induziert worden.

Durch den hier wiedergegebenen Fallverlauf mit histologischer Bestätigung des unmittelbaren Ansprechens auf homöopathische Mittelgabe ergeben sich weitere Hinweise auf eine eigenständige antitumoröse Wirkung homöopathischer Therapie und damit einen "Zusatznutzen der Homöopathie in der Onkologie" (Wurster und Frass) [20]. Sie kann sich nach als "ein wichtiger Bestandteil einer integrativen Medizin" (Matthiessen) erweisen [21].
Zur weiteren Evaluation der homöopathischen Therapie und Wirkung bei Tumor-Erkrankungen sind weitere Studien erforderlich, welche sich auf objektive (Tumor)Verlaufsparameter stützen sollten.

Es versteht sich von selbst, dass jegliche rein homöopathische Tumortherapie nur unter engmaschiger Erfolgskontrolle angewandt werden darf. Gegebenenfalls muss eine Umstellung auf, beziehungsweise Ergänzung durch, konventionelle Radio-Chemotherapie erfolgen. Es darf definitiv nicht zu einer ungerechtfertigten Verzögerung von etablierten, durch Evidenz gesicherten Therapien kommen.

Es kann somit davon ausgegangen werden, dass bei strikter Einhaltung obiger Behandlungsprinzipien nicht nur die begleitende, sondern auch die alleinige homöopathische Tumortherapie sowohl zulässig als auch sinnvoll ist.

\section{Disclosure Statement}

Die Autoren erklären, dass kein Interessenkonflikt besteht.

\section{Literatur}

1 Schroyens F, editor. Synthesis: Repertorium homoeopathicum syntheticum. Edition 9.1. Greifenberg: Hahnemann Institut; 2005

2 Schroyens F, editor. Radar 10.5 für Windows. Gent: Archibel; 2011.

3 Hümmer H. Evidence-based Medicine und Homöopathie. In: Bitschnau M, Drähne A, editors. Homöopathie in der Frauenheilkunde. München, Jena: Urban \& Fischer; 2007. pp. 43-8.

4 Mathie RT, Ramparsad N, Legg LA, Clausen J, Moss S, Davidson JR, et al. Randomised, double-blind, placebo-controlled trials of non-individualised homeopathic treatment: systematic review and meta-analysis. Syst Rev. 2017 Mar;6(1):63.

5 Walach H. Die Europäischen Wissenschaftsakademien und die Homöopathie. 2017. Available from: www.homöopathie-forschung.info/easac/

6 Lüdtke R, Rutten AL. The conclusions on the effectiveness of homeopathy highly depend on the set of analyzed trials. J Clin Epidemiol. 2008 Dec;61(12):1197-204.

7 Shang A, Huwiler-Müntener K, Nartey L, Jüni P, Dörig S, Sterne JA, et al. Are the clinical effects of homeopathy placebo effects? Comparative study of placebo-controlled trials of homeopathy and allopathy. Lancet. 2005 Aug-Sep;366(9487):726-32.
8 Hümmer H, Pachmann K, Pachmann U. Adjuvante homöopathische Therapie bei konventionell behandeltem Mammakarzinom. Verlaufs- und Therapiekontrolle mittels quantitativer Bestimmung im Blut zirkulierender epithelantigen-positiver Zellen. AHZ. 2005;250:127-33.

9 Lehrke P, Quak T, Wurster J. Adjuvante Homöopathie in der Onkologie. München: Elsevier; 2018.

10 Payrhuber D. Homöopathie und Krebs: Möglichkeiten zur Heilung. Salzburg: Narayana Verlag; 2006.

11 Horning SJ, Rosenberg SA. The natural history of initially untreated low-grade nonHodgkin's lymphomas. N Engl J Med. 1984 Dec;311(23):1471-5.

12 Potts DA, Fromm JR, Gopal AK, Cassaday RD. Spontaneous Remission of an Untreated, MYC and BCL2 Coexpressing, High-Grade B-Cell Lymphoma: A Case Report and Literature Review. Case Rep Hematol. 2017;2017: 2676254.

13 Grem JL, Hafez GR, Brandenburg JH, Carbone PP. Spontaneous remission in diffuse large cell lymphoma. Cancer. 1986 May; 57(10):2042-4.

14 McCabe MG, Hook CE, Burke GA. Spontaneous regression of an EBV-associated monoclonal large $\mathrm{B}$ cell proliferation in the mastoid of a young child following surgical biopsy. Pediatr Blood Cancer. 2008 Oct;51(4):557-9.

15 Abe R, Ogawa K, Maruyama Y, Nakamura N, Abe M. Spontaneous regression of diffuse large B-cell lymphoma harbouring Epstein-Barr virus: a case report and review of the literature. J Clin Exp Hematop. 2007 Apr;47(1):23-6.
16 Karnad AB, Jaffar A, Lands RH. Spontaneous regression of acquired immune deficiency syndrome-related, high-grade, extranodal non-Hodgkin's lymphoma. Cancer. 1992 Apr;69(7):1856-7.

17 Kumamoto M, Nakamine H, Hara T, Yokoya $\mathrm{Y}$, Kawai J, Ito H, et al. Spontaneous complete regression of high grade non-Hodgkin's lymphoma. Morphologic, immunohistochemical, and gene amplification analyses. Cancer. 1994 Dec;74(11):3023-8.

18 Marzotto M, Olioso D, Brizzi M, Tononi P, Cristofoletti M, Bellavite P. Extreme sensitivity of gene expression in human SH-SY5Y neurocytes to ultra-low doses of Gelsemium sempervirens. BMC Complement Altern Med. 2014 Mar;14(1):104.

19 Kappauf H, Büschel G, Kaiser G, Gallmeier WM. Dokumentation von Spontanremissionen bei Krebserkrankungen durch die Nürnberger Arbeitsgruppe Biologische Krebstherapie - Auswertung von Laienberichten. In: Heim ME, Schwarz R, editors. Spontanremissionen in der Onkologie. Stuttgart: Schattauer; 1998. p. 57-65

20 Wurster J, Frass M. Replik auf den Leserbrief von Herrn Dr. Norbert Aust et al. zu „Zusatznutzen der Homöopathie in der Onkologie“. Dtsch Z Onkol. 2018;50(04):193-193.

21 Matthiessen P. Homöopathie und intellektuelle Redlichkeit - Eine Stellungnahme. Dtsch Z Onkol. 2018;50(04):172-7. 